\title{
HYBRID SKIN DESIGN OF THE TRANSITION REGION BETWEEN MORPHING WING AND FIXED WING
}

\author{
Martin Radestock* \\ Institute of Composite Structures \\ and Adaptive Systems \\ German Aerospace Center (DLR) \\ Lilienthalplatz 7 \\ 38108 Braunschweig, Germany \\ Email: Martin.Radestock@dlr.de
}

\author{
Alexander Falken \\ INVENT Innovative Verbundwerkstoffe \\ Realisation und Vermarktung \\ neuer Technologien GmbH \\ Christian-Pommer-Straße 34 \\ 38112 Braunschweig, Germany
}

\author{
Johannes Riemenschneider, \\ Markus Kintscher \\ Institute of Composite Structures \\ and Adaptive Systems \\ German Aerospace Center (DLR) \\ Lilienthalplatz 7 \\ 38108 Braunschweig, Germany
}

\section{ABSTRACT}

The adaptation of a wing contour is important for most aircraft, because of the different flight states. That's why an enormous number of mechanisms exists and reaches from conventional slats and flaps to morphing mechanisms, which are integrated in the wing. Especially integrated mechanisms reduce the number of gaps at the wing skin and produce less turbulent flow. However these concepts are located at a certain section of the wing. This leads to morphing and fixed wing sections, which are located next to each other. Commonly, the transition between these sections is not designed or a wing fence is used. If the transition is not designed, the wing has a step with an activated morphing mechanism and that produces additional vortices. A new skin design will be presented in order to smooth the contour between a fixed wing and a morphing wing. Here the transition between a droop nose and a fixed wing is considered. The skin material is a mix of ethylene propylene diene monomer rubber and glass-fiber reinforced plastic. The rubber is the baseline material, while the glass- fiber is added as stripes in chord-wise direction. In span-wise direction the glass fiber is connected with the rubber. The rubber carries the loads in span-wise direction and reduces the required actuation force. The glass fiber stiffens the skin locally in chord wise direction and keeps the basic contour of the skin.

\footnotetext{
*Address all correspondence to this author.
}

Some geometrical parameters within the skin layup can be varied to change the transition along the span or to reduce the maximum strain within the skin. The local strain maximum is a result of the material transition with different modules. One design of a leading edge was manufactured with an existing mold and it has a span of $200 \mathrm{~mm}$. There are two essential aspects from a structural point of view. One is a nearly continuous deformation along the span and the second is the maximum strain in the rubber. Both aspects are investigated in an experiment and the results are compared with a simulation model. The results show a reliable concept and its numerical model, which will be assigned to a full scale demonstrator. This demonstrator will have a span of $1000 \mathrm{~mm}$ and will show the smooth skin transition between a droop nose and a fixed wing.

\section{NOMENCLATURE}

$u, v, w$ Displacement in $\mathrm{x}, \mathrm{y}, \mathrm{z}$ direction of coordinate system, respectively

$L_{\text {outer }}, L_{\text {inner }}$ Length of the GFRP stripes at the outer or inner shape contour

$t_{G F R P}$ Thickness of the GFRP stripes 


\section{INTRODUCTION}

For every aircraft the adaption of the wing shape is crucial. So, the velocity at take-off and landing can be less than in cruise flight. Mostly slats and flaps are used to reach the required shape geometry in order to ensure enough lift. There are also a lot of other concepts to realize the shape adaption. The work of Barabarino and Vasista summarizes different morphing types at the fixed wing of aircraft $[1,2]$. The technologies reach from planform alteration like sweep morphing up to airfoil adjustment like camber morphing. Especially, the adjustment of an airfoil is often an integrated concept and is limited to a certain span section of the wing. In cruise flight the wing has a continuous geometry in span-wise direction, while the morphed configuration produces a step at its span-wise edges. Often this step is not designed or a wing fence is used. This produces vortices, which can be reduced when the transition between a fixed wing section and a morphing section is smooth [3]. In contrast to the morphing section the skin in the transition region should have different materials. The main difference is the distortion condition. In a camber morphing section the major distortion is in chord direction while the major distortion in the transition section is in chord and span-wise direction. Also, the deformation of the skin in the transition region requires additional forces from the camber morphing actuation. Therefore, it is useful to focus on soft skin. The example of this paper is situated at the leading edge of a wing from a short range aircraft. The camber morphing at this wing is realized with a droop nose and it is analog to the work of Kintscher [4]. The presented skin for the transition region is a material mix, where the major component is ethylene propylene diene monomer (EPDM) rubber and the minor component is glass fiber reinforced plastics (GFRP). Due to the arrangement of the GFRP in the skin, it is possible to influence the stiffness of the skin. That allows an adaption of the outer skin contour during the deformation process of the morphing section.

The first part of this paper presents the general concept of the skin. This part describes the dimensions of the skin, the wing shapes and the preparation of an experimental demonstrator, which is scaled down. The second part approaches the simulation model of the skin. That includes the simplification of the demonstrator, the boundary conditions and the results of the finite element analysis (FEA). The third part describes the experiment with a manufactured skin. The focus is the deformation and the strain distribution of the skin, which will be presented and compared to the simulation model. All results show a reliable concept for the transition region of the skin from a structural point of view. This work is the baseline for a future full scale demonstrator with an approximated camber variation mechanism.

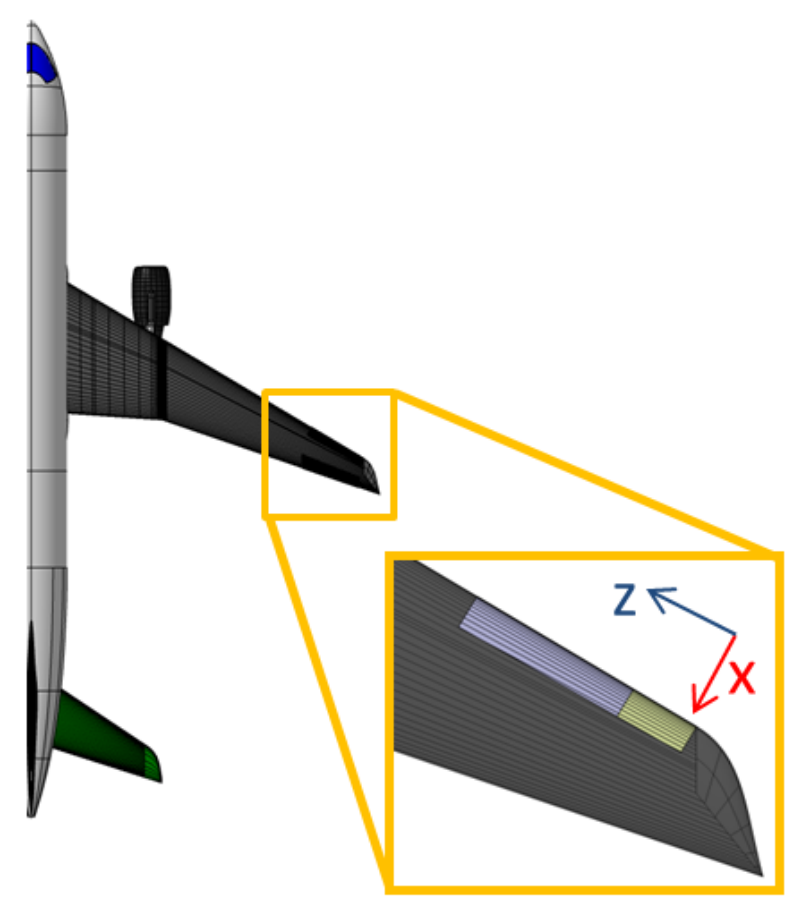

FIGURE 1. PLAIN VIEW OF THE WING WITH A SECTION FOR CAMBER MORPHING (LIGHT BLUE) AND A SECTION FOR THE TRANSITION SKIN (YELLOW)

\section{CONCEPT DESCRIPTION}

The aircraft used to demonstrate this concept is from an aerodynamic wing optimization, which was developed by the German Aerospace Center and Airbus [5]. In figure 1 the half model of the aircraft is shown. This model is similar to current short range aircraft like A320. The zoom in figure 1 shows the region where the furthest slat is located. For this concept this section is divided in two sections. The light blue part represents the section of the camber variation concept, which is a droop nose. This section has a span of approximately 2.5 meters. The yellow part is the section for the transition skin. The span of this section is one meter.

A framework is used to achieve a target shape for the droop nose concept. In the work of Kintscher the geometrical design tool is summarized [4]. The resulting geometry for a droop variation is shown in figure 2 . The advantage of this tool is the shape generation with a constant length of the leading edge geometry. The continuous line is the shape of the fixed wing section and the dashed line is the droop shape for the morphing section. The droop angle is determined with the chord lines of the shape. Therefore, 


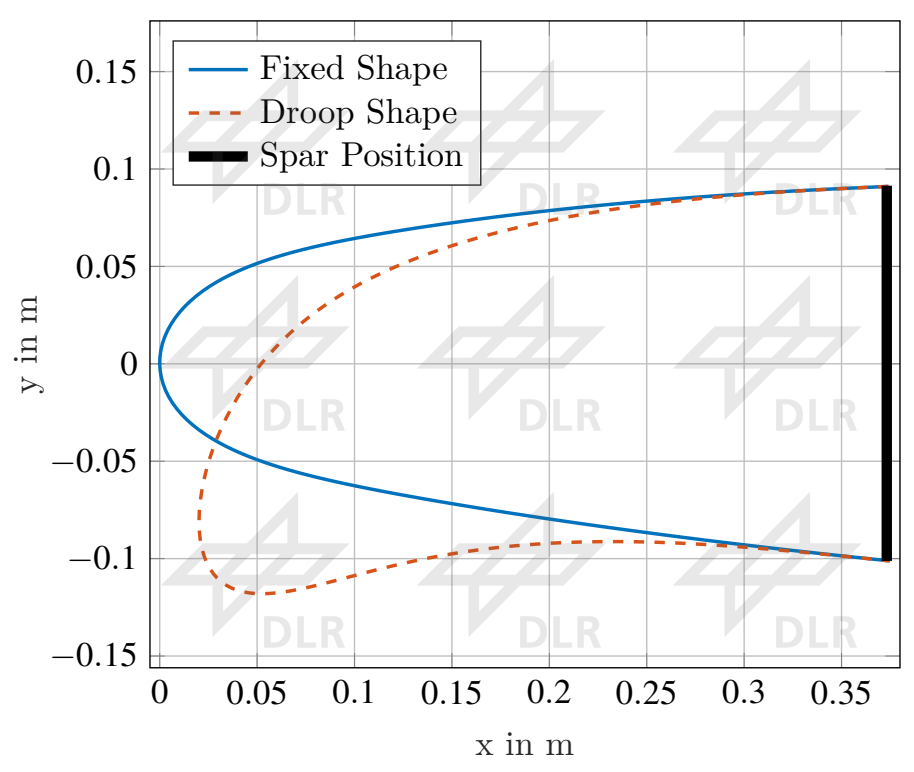

FIGURE 2. SHAPE OF THE FIXED WING SECTION (CONTINUOUS) AND SHAPE OF THE MORPHING SECTION (DASHED) IN PLAIN VIEW

two intersections are determined. The first one is the intersection between chord line and shape, which is equal to the point at the nose. The second intersection is between chord line and spar. For one shape the two intersections are connected with a line. The angle between the resulting lines is determined and it is approximately 11 degrees.

Two simplifications are assumed for the new skin concept. The first simplification is the reduction of the span. It is reduced from one meter to 200 millimeters, because an existing mold is utilized. The other dimensions of this mold are similar to the leading edge. The second simplification neglects the morphing mechanism in the experimental setup. Only one point for displacement transmission determine the distortion of the skin and is indicated in figure 3 with v.

The connections to the spar and the span-wise fixation are realized with screws and the drill holes are indicated in figure 3. The skin is under compression at the screwing. It is obvious to avoid EPDM in that part of the skin. At the interfaces the skin is made of pure GFRP layers. The pure GFRP region of the skin has a span of 40 millimeters for the span-wise fixation. In chord direction the pure GFRP region has a length of 140 millimeters for the spars on the lower and upper side of the skin.

The skin concept uses a mix of EPDM as baseline material and additional GFRP stripes as stiffeners. Figure 4 shows the assembly of both components for the whole skin.



FIGURE 3. CAD MODEL OF THE PRINCIPAL EXPERIMENTAL SETUP WITH SKIN (LIGHT BLUE), FIXATIONS (GREY) AND INDICATED DISPLACEMENT TRANSMISSION POINT (ORANGE)

Here it is possible to vary the number, the width or the layup of the GFRP stripes. That allows an adaption of the stiffness of the skin. A variation of the GFRP thickness in chord direction is neglected for the first skin design. The section A-A in figure 5 represents the setup of the skin for the experiment and the simulation. The thickness of each GFRP stripe is set to one millimeter. Also, the length of the first GFRP stripes are different at the outer and inner skin contour, which are designated with $L_{\text {outer } 1}$ and $L_{\text {inner } 1}$. The result of the different length is an overlap of the following GFRP stripes at the outer and inner contour. Basically it is possible to vary the length of each GFRP stripe, but here the length is set to $25 \mathrm{~mm}$. Also, the spaces between the GFRP stripes are constant with 10 millimeters. The pure GFRP stacking in figure 5 represents the skin connection for the span-wise fixation.

In preparation of the manufacturing the model has two additional EPDM layers on the outside and on the inside of the skin contour. These two layers are not shown in figure 4 , because they reduce the depiction of the concept. The simulation results as well as the experimental results include these two layers.

The GFRP is a prepreg with glass fiber from Suter Kunstoffe AG company and a resin from Gurit company $[6,7]$. The thickness of one GFRP layer is $0.25 \mathrm{~mm}$ and over- 


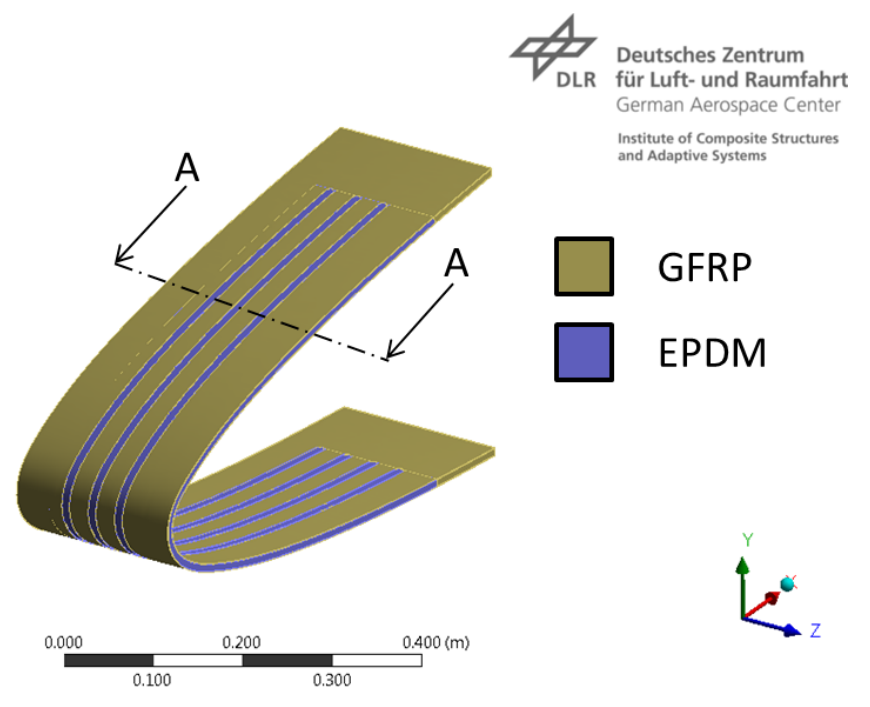

FIGURE 4. MODEL OF THE SKIN WITH EPDM AND GFRP STRIPES

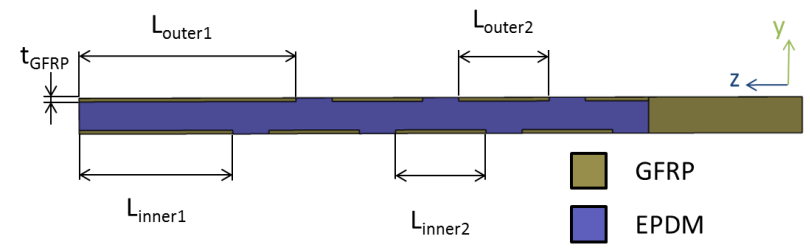

FIGURE 5. SECTION A-A OF THE SKIN WITH ARRANGEMENT OF GFRP STRIPES

all thickness of the GFRP stripes are one millimeter. The fibers are oriented in chord direction as zero degree. The layup for each GFRP stripe is out of four zero degree layers. The EPDM is a rubber from Kraiburg company with the label AA6CFZ [8]. It is provided prepreg, but has a thickness of 0.5 millimeters. The datasheets for the EPDM and the GFRP provide the general material data for the simulation models.

\section{SIMULATION MODEL}

The FEA is done with the software Ansys ${ }^{\circledR}$ Workbench $^{\mathrm{TM}}$. The elements in the simulation are linear solid elements with 8 nodes. In order to reduce simulation costs only the skin is simulated. The spar and the span-wise fixation are replaced by boundary conditions. In case of the relatively high deformation compared to the dimensions, the FEA uses the nonlinear calculation.
TABLE 1. List of the material properties for the simulation

\begin{tabular}{cll}
\hline Property & GFRP & EPDM \\
\hline Young's modulus in x direction [MPa] & $19 \mathrm{e} 3$ & 1.6 \\
Young's modulus in y direction [MPa] & $7 \mathrm{e} 3$ & 1.6 \\
Young's modulus in z direction [MPa] & $7 \mathrm{e} 3$ & 1.6 \\
Poisson's ratio xy in [-] & 0.26 & 0.45 \\
Poisson's ratio yz in [-] & 0.26 & 0.45 \\
Poisson's ratio xz in [-] & 0.26 & 0.45 \\
Shear modulus xy in [MPa] & $5.6 \mathrm{e} 3$ & 0.552 \\
Shear modulus yz in [MPa] & $4.7 \mathrm{e} 3$ & 0.552 \\
Shear modulus xz in [MPa] & $5.6 \mathrm{e} 3$ & 0.552 \\
Density in $\left[\mathrm{kg} / \mathrm{m}^{3}\right]$ & 1950 & 1040 \\
\hline
\end{tabular}

The material properties of the simulation are listed in table 1. The material model for the EPDM is isotropic and the material model for the GFRP is orthogonal-anisotropic. Both materials are simulated with linear elastic models. The EPDM is in general a hyper-elastic material, but the expected strain is less than $10 \%$. In the datasheet [8] three data points are given for the Young's modulus at 50\%, $100 \%$ and $200 \%$ strain. The value at $50 \%$ strain is used as Young's modulus of the isotropic model. The properties of the GFRP are out of the datasheets $[6,7]$. In these documents some relevant material data are not available. Therefore, the missing properties are from literature [9].

All three boundary conditions like applied displacement $\mathrm{v}$ and the two fixations are shown in figure 6 . They are realized with the direct FE description of nodal displacements. The fixation in span-wise direction and the fixation at the spar are set to zero for each direction in the coordinate system.

The third boundary condition is the displacement, which is already indicated in figure 3. The applied displacements are node displacements with 14 millimeters. It is obvious that the 14 millimeters do not represent the whole droop deformation in figure 2 , but the skin with a span of 200 millimeters does not replace the whole transition region.

The displacement is a result of a relative consideration of the complete skin. Therefore, the shapes from figure 2 are set in a distance of one meter. Afterwards the nose points of the shapes are connected with a spline. This spline is perpendicular to the $\mathrm{x}-\mathrm{y}$ plane at the endpoints. Finally, 



FIGURE 6. POSITION OF THE BOUNDARY CONDITIONS OF THE SIMULATION MODEL (PURPLE)



FIGURE 7. DESIGN OF THE TRANSITION SURFACE WITH THE NOSE SPLINE(DASHED) AND SECTIONS (DOTTED) WITH A DISTANCE OF 200 MILLIMETERS

a surface is generated with the spline as guided line. The calculation of the spline geometry and the surface is done with CATIA ${ }^{\mathrm{TM}}$. The result is shown in figure 7 .

The surface is divided in five sections with a span of 200 millimeters. It is assumed that the length of each section is constant. Now, the sections are distributed in 100 equidistant points and numbered consecutively for each section. Each point moves in the $\mathrm{x}-\mathrm{y}$ plane and the absolute distance is calculated. The relative motion between the adjacent sections are relevant for the simulated skin. Therefore, the delta of the absolute distances between adjacent points is calculated. Adjacent points have the same number in nearby sections, e.g. point 23 in section 2 is compared with point 23 in section 3 . The maximum of all relative displacements is 14 millimeters. Also, the position of the Maximum along the chord direction is at the same position as indicated in figure 6 .
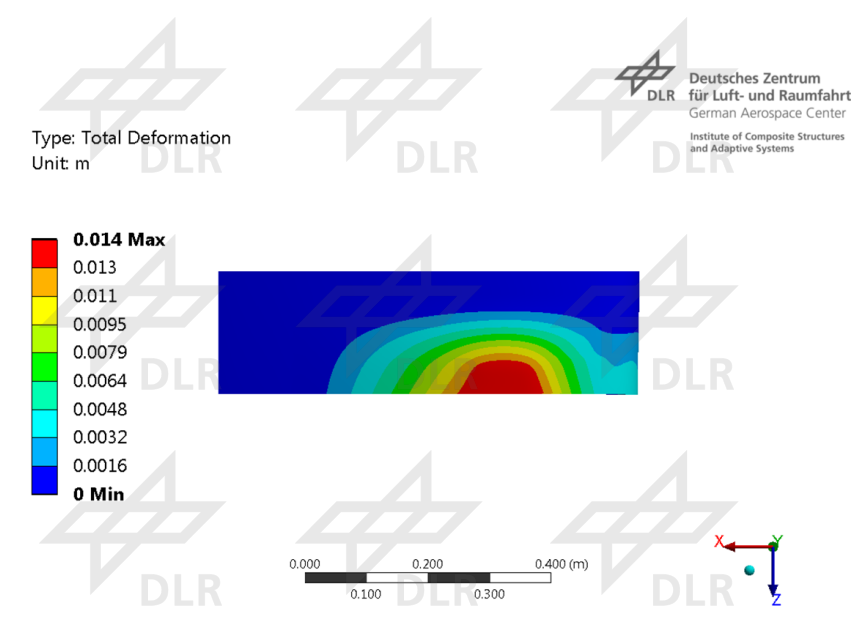

FIGURE 8. DEFORMATION OF SKIN ON THE LOWER SIDE FROM THE SIMULATION MODEL

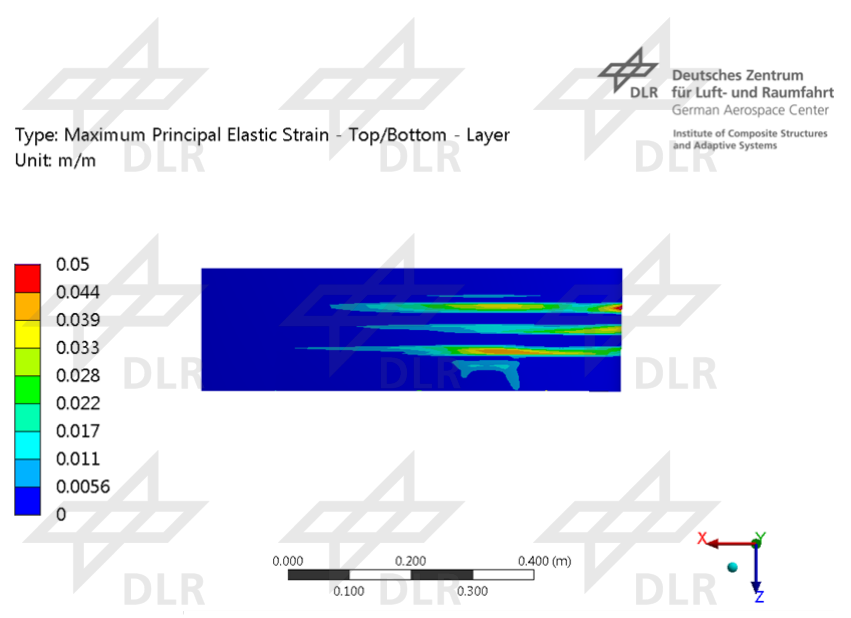

FIGURE 9. STRAIN ON THE LOWER SIDE OF THE SKIN FROM THE SIMULATION MODEL

The main objective of the simulation is the analysis of the deformation and the calculation of the strain. The maximum strain is relevant, because the EPDM can be peeled off from GFRP. The bonding between GFRP and EPDM is only a glued connection. One challenge for the experiment is the measurement of the strain. The maximum strain occurs in the EPDM, but in case of the low Young's modulus, strain gauges are not applicable. Alternatively, an optical measurement is used. For comparison the strain only on the outer skin contour is evaluated in the simulation. If the strain is similar to the experiment, then the simulation should give a good approximation of the strain in the skin. The critical position of the skin is expected on the lower 
side of the skin. The displacement is applied at one side and so the evaluation of the deformation and the strain will be essential at that side.

Figure 8 shows the deformation result from the simulation at the lower skin side. The deformation is along the whole span and it is not local at the first GFRP stripe where the displacement is applied. Even with the stiffer GFRP the EPDM transfers the displacement over the whole span. It is possible, because the stiffer GFRP has a small thickness compared to the EPDM. So, next to the material properties the dimensions of each material is relevant for this concept. If the EPDM thickness will decrease, then the skin is getting compliant. A transmission of a deflection is getting less effective, because the strain in the EPDM will increase and the deformation is more local.

In order to analyze the compliance figure 9 shows the strain of the skin. There are three stripes with higher strain. These are the gaps out of EPDM between the GFRP stripes. The maximum strain is derived between the GFRP stripe where the displacement is applied and the adjacent GFRP stripe, which is labeled as first EPDM gap. At the second gap the strain has the lowest values of all three gaps. At that position the skin has the highest flexibility, because adjacent GFRP stripes are not influenced by boundary conditions. The third gap has higher strains than the second gap, but the values are lower than at the first gap. Here the boundary condition of the span-wise fixation has an influence and the strain increases.

\section{EXPERIMENTAL INVESTIGATION}

In case of the soft material EPDM it is challenging to measure the strain in the common way with strain gauges. An alternative is the measurement of the skin surface with an optical digital image correlation (DIC) system. That system can measure without a direct contact, but cannot analyze the strain within the skin. So, it is not possible to identify the maximum strain when it is not at the surface. ARAMIS is the name of the optical DIC system and is provided by the company GOM GmbH. The system uses a stereographic camera system, which evaluates a surface. The overall setup is shown in figure 10. Also, the presented results are limited to the lower side of the skin, because the maximum strain and the maximum deformation are expected at this side.

The design of the span-wise fixation is a bit different from the CAD model in figure 3, but fulfill the same boundary condition. The applied displacement is realized with a screw, which goes through a fixed nut and pushes the skin. Figure 11 shows an aluminum profile next to the skin, where the displacement mechanism is connected. The zoomed image shows the interface with a replaced profile in order to



FIGURE 10. EXPERIMENTAL SETUP OF THE SKIN WITH DIC SYSTEM

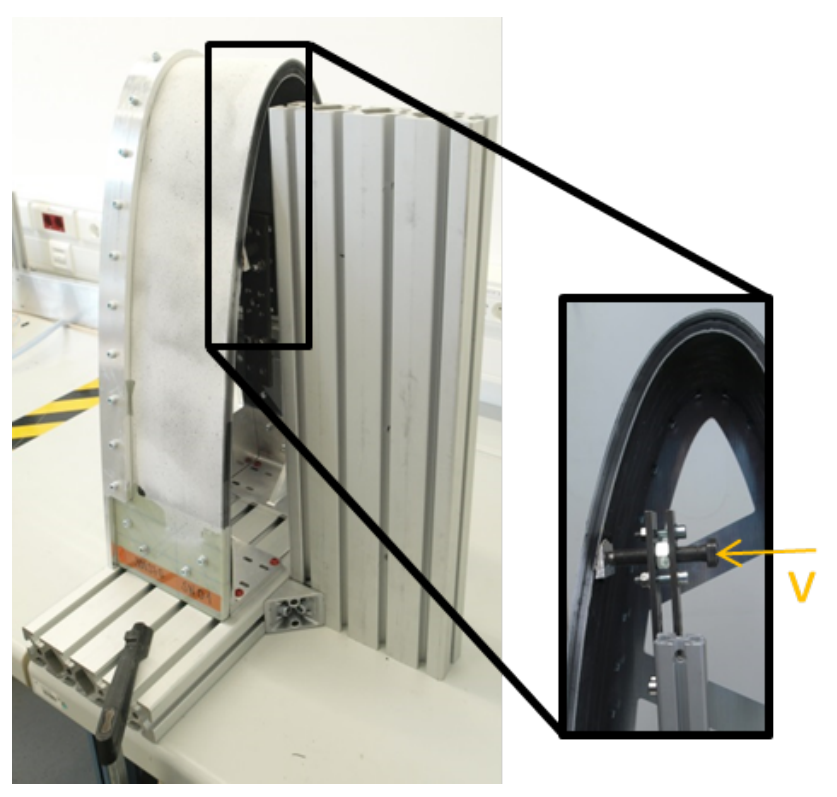

FIGURE 11. INTERFACE OF THE APPLIED DISPLACEMENT AND ZOOMED IMAGE WITH A SIMPLIFICATION OF THE INTERFACE WITH A SCREW FOR DEPICTION

visualize the mechanism. For the experiment the massive aluminum profile is used in order to prevent the bending of the displacement interface.

In case of a deformation the camera systems records images of the lower skin side. In figure 10 the skin has a white surface. This white paint has additional small black dots, which are sprayed with a varnish. The black-white pattern is stochastic. That is essential for the image correlation, 


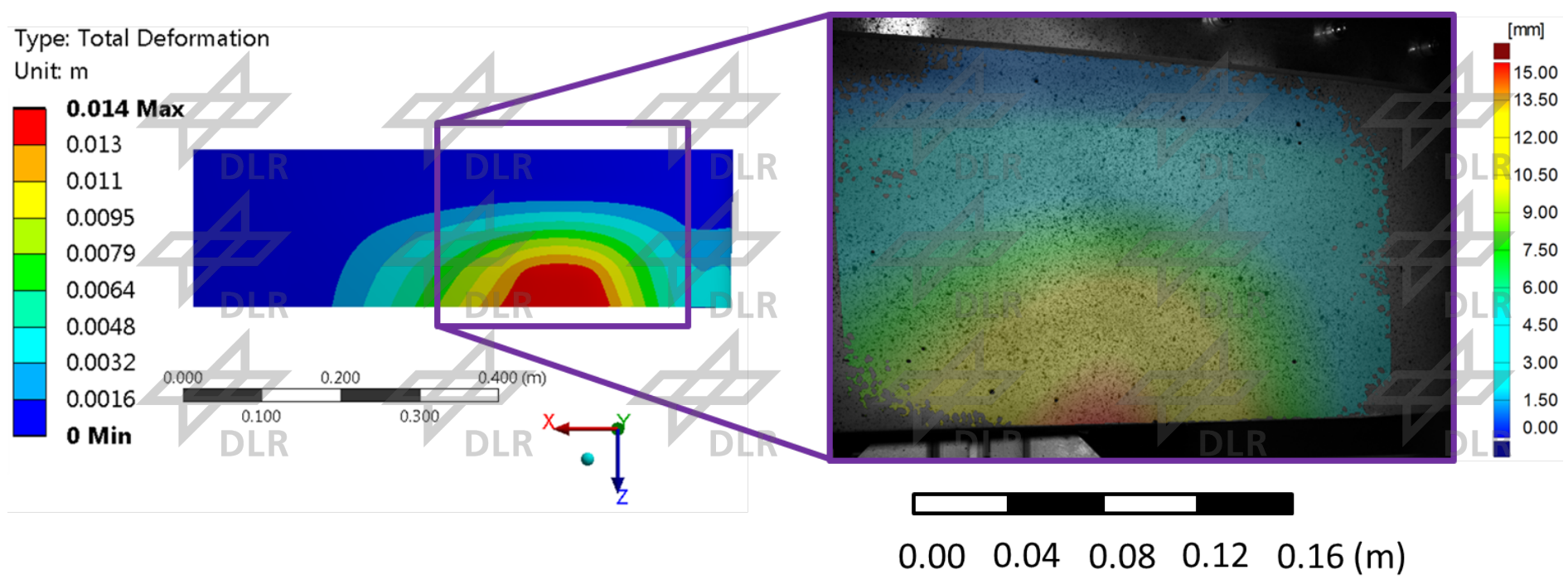

FIGURE 12. ABSOLUTE DISPLACEMENT OF THE SKIN ON THE LOWER SIDE IN THE SIMULATION WITH THE EXPERIMENTAL RESULTS AS A SECTION

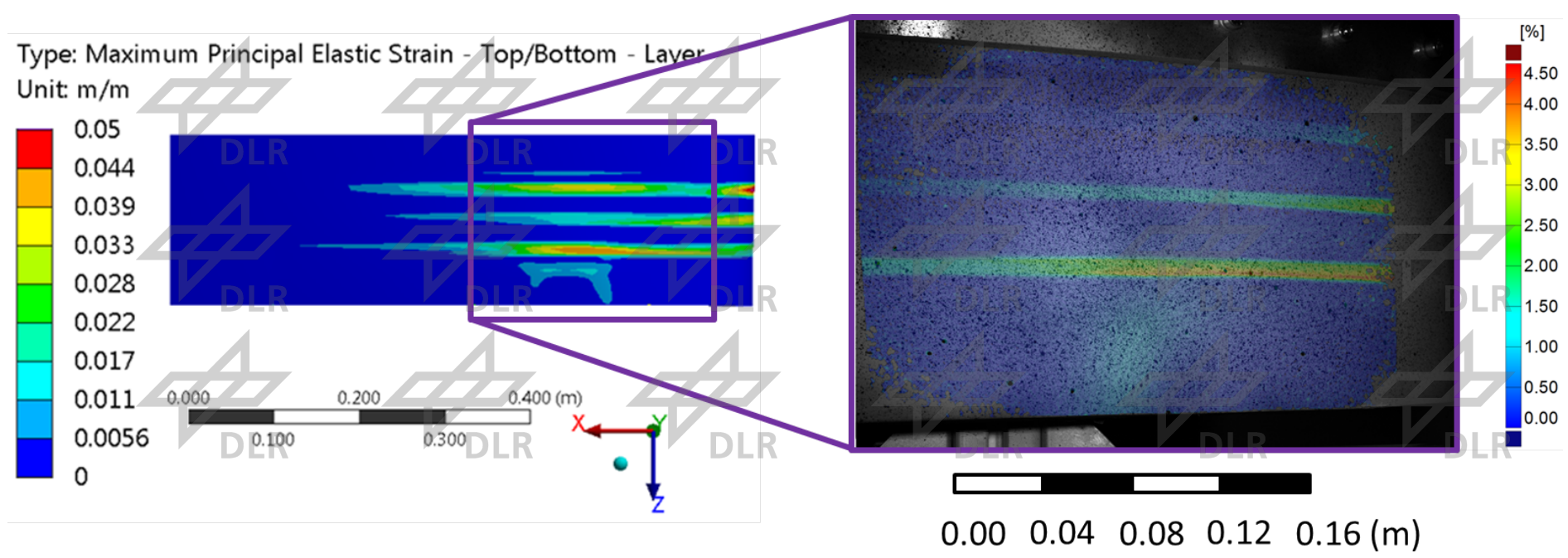

FIGURE 13. STRAIN ON THE LOWER SIDE OF THE SKIN IN THE SIMULATION WITH THE EXPERIMENTAL RESULTS AS A SECTION

because the system divides the captured images into small facets. Each facet has a unique black-white pattern and can be tracked by the system. If the pattern is distorted, than the systems can evaluate the strain of the surface.

At the beginning of the experiment two images were made. The first image is the reference image and the second image is done a few seconds later without a change at the skin. The calculated divergence is an indicator for the accuracy of the system and the pattern. In case of the absolute deformation the divergence is approximately 0.8 millimeters. If the strain is considered, the divergence is approximately $0.3 \%$. The accuracy of the strain depends on regarded area in the measurement. The strain at edges cannot be evaluated, because the systems interpolate the strain between different facets. At the edges the number of facets is too low and the results can be incorrect. 
Figure 12 shows the result of the displacement in the experiment as a section and in the simulation. The deformation in the experiment is not located at a certain area and is distributed over the whole span. Also, it is not possible to identify the gaps between the GFRP stripes, because the colors for the deformation are distributed continuously.

In order to identify the location of the gaps between the GFRP stripes the strain has to be analyzed. Figure 13 shows the strain on the lower side in the experiment and in the simulation. The maximum measured strain is $4.5 \%$. This value is low compared to the range of the strain from the datasheet of the EPDM. Three EPDM gaps are identified, which is similar to the simulation. The maximum strain is on the first EPDM gap where the displacement is applied. The strain in the gaps decreases when they are located closer to the span-wise fixation.

Compared to the datasheet the measured strain is low with $4.5 \%$. Another result of the measurement is the decrease of the strain if the gap between GFRP stripes is located closer to the span-wise fixation. That is already expected from the simulation.

\section{COMPARISON OF SIMULATION AND EXPERIMENT}

The comparison of the experiment with the simulation can be split into the two relevant results deformation and strain. The deformation in simulation and experiment are qualitatively equal. Both results show a deformation in span-wise direction and the maximum at the applied displacement. A difference is visible at the size of the applied displacement. In the experiment the area with the maximum deformation (red region) has an approximated length in $\mathrm{x}$ direction of 0.04 meter while in the simulation the length is nearly 0.1 meter. The difference is a result of the boundary condition in the simulation. The applied displacement is realized with a direct nodal formulation of the displacement. In the experiment the material is flexible and the skin can deform when the displacement is applied with the screw. Hence, the region of the maximum displacement is smaller in the experiment.

While comparing the strain the general tendency is the same, but the strain in the experiment is lower than in the simulation. That means the EPDM in the experiment does not deform as much as in the simulation. This can be an effect of the material data from datasheets. The EPDM is an hyper-elastic material and cannot be characterized with 3 data points in strain from the datasheet. Especially, when the data points are at 50\%, 100\% and 200\% strain. As a result of that comparison an experiment for characterizing the EPDM is set on. The result of a tensile test will be used for a hyper-elastic material model instead of a linear isotropic material model in upcoming simulations.

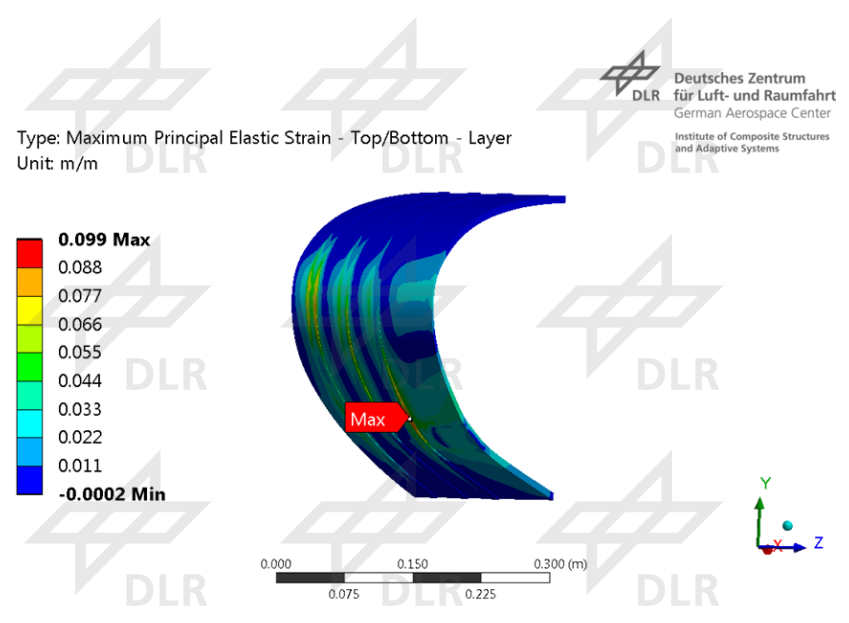

FIGURE 14. STRAIN OF THE EPDM BODY IN THE MIDDLE PART OF THE SKIN WITHOUT GFRP AND WITHOUT FIXATIONS

Another difference of the strains between simulation and experiment can be the fact of the strain calculation. In the simulation the calculated strain depends on the element size. Especially, the borders of material shift are important in the simulation. Higher strains can occur at material transition with different stiffness. Also, the strain in the experiment is interpolated between different facets of the image. If the pattern quality varies over the skin and the considered region is small, the strain can change slightly and locally.

In general, the overall results show acceptable estimation of the simulation for the manufactured skin. As the simulation gives a strain estimation of the skin at the inside where the optical system cannot measure. The maximum strain amounts approximately $10 \%$ and is located at the border between GFRP and EPDM on the inside of the skin. Figure 14 shows the location of the maximum strain, which is near to the applied displacement. The figure shows only the EPDM, which is in the middle of the skin and which connects the GFRP stripes. As a result the concept is reliable for future work, because the estimated strains in the simulations are lower than the limits of EPDM and GFRP.

\section{CONCLUSION}

The presented concept is a new approach for a skin, which smooth the aerodynamic surface between a morphing mechanism and a fixed wing. The concept is applied for skin between a camber morphing leading edge and the fixed wing near to the wing tip. The first step was a reduction of the model in span from 1000 millimeter to 200 
millimeters, because an existing mold is used. The chord and thickness are still full scale. The skin is a material mix of ethylene propylene diene monomer rubber and glass-fiber reinforced plastic. Next to a simulation model an experiment was set up. The main objective was the analyses of strain and deformation behavior and a validation between the simulation and the experimental model. The results in strain and deformation vary slightly between the experiment and the simulation, but in general the results are close to each other. So, the simulation validate sufficient the skin concept. Also, the limits of the material are not exceeded, either in the experiment or in the simulation. Therefore, the presented concept is reliable for future work, where a full scale demonstrator is manufactured including a morphing mechanism as actuation stations.

\section{ACKNOWLEDGMENT}

Thanks go to Johannes Achleitner (chair of lightweight structures) and Jan Krollmann (chair of carbon composites) from the technical university of Munich for their ideas and feedback during the project. Thanks go to the Federal Ministry of Economic Affairs and Energy for partially supporting the work as a part of its aviation research program under contract LUFOV2-790-305.

\section{REFERENCES}

[1] Barbarino, S. et al., 2011. "A review of morphing aircraft". Journal of Intelligent Material Systems and Structures, 22(9), August, pp. 823-877.

[2] Vasista, S., Tong, L., and Wong, K., 2012. "Realization of morphing wings: A multidisciplinary challenge". Journal of Aircraft, 49(1), January, pp. 11-28.

[3] Kota, S. et al., 2009. "Mission adaptive compliant wingdesign, fabrication and flight test". RTO Applied Vehicle Technology Panel (AVT) Symposium, April.

[4] Kintscher, M. et al., 2011. "Design of a smart leading edge device for low speed wind tunnel tests in the european project sade". International Journal of Structural Integrity, 2(4), pp. 383-405.

[5] Dargel, G. et al., 2002. "Aerodynamische flügelauslegung mit multifunktionalen steuerflächen". In DGLR Jahrbuch 2002, A. Editor and B. Editor, eds., Vol. 1, Deutsche Gesellschaft fuer Luft- und Raumfahrt, DGLR, p. 1605.

[6] Schillinger, 1999. GLASS FILAMENT FABRICS for PLASTICS REINFORCEMENT. Suter Kunstoffe AG, 3312 Fraubrunnen Switzerland. See also URL: www.swiss-composite.ch/pdf/t-Glas-296g-92626-Atlase.pdf.

[7] Gurit, 2018. General Data sheet EH 250 ,EP
112. Gurit Services AG, Thurgauerstrasse 54, 8050 Zurich , Switzerland. See also URL: www.gurit.com/-/media/Gurit/Datasheets/eh-250.pdf.

[8] Schaube, J., 2013. TECHNICAL DATA SHEET AA6CFZ. Gummiwerk KRAIBURG GmbH and Co. KG, Teplitzer Str. 20, 84478 Waldkraiburg, Germany.

[9] Schürmann, H., 2007. Konstruieren mit FaserKunststoff-Verbunden. Springer, Berlin Heidelberg New York. 